\title{
About the decay of the neutral Kaon into three Pions in the book: The Feynman Lectures on Physics
}

\author{
J.D. Bulnes ${ }^{* 1}$, A.B. Calado ${ }^{1}$ \\ ${ }^{1}$ Universidade Federal do Amapá, Departamento de Ciências Exatas e Tecnologia, Macapá, AP, Brasil.
}

Received on May 17, 2021. Revised on June 19, 2021. Accepted on June 21, 2021.

In this article we present a mathematical solution that extends and explores the solution found in chapter 11 of the book "Quantum Mechanics", from "The Feynman Lectures on Physics", about the neutral Kaon. The extension is built within the context of the $C P$ symmetry for the two neutral Kaon decays considered in this chapter: (i) In two Pions, starting in the $\left|K_{1}\right\rangle$ state; (ii) In three Pions, from the $\left|K_{2}\right\rangle$ state. We found two mathematical expressions that allow to accommodate, separately, each one of the mean lifetime for these decays, unlike the solution given by Feynman that allows to accommodate only one of these mean lifetime. The extended solution is approximate because a small break in CPT symmetry is assumed in these decays; however, it has the advantage of avoiding the colloquially commented situation by Feynman of remaining "forever" from the neutral Kaon in the $\left|K_{2}\right\rangle$ state in the situation in which the only possible decay channel was in two Pions.

keywords: Neutral $K$ meson, $C P$ and $C P T$ symmetry, Decay of the neutral Kaon into three Pions.

\section{Introduction}

Until the mid-1960s, many physicists accepted that the so-called $C P$ symmetry [1] was preserved in the processes in which unstable quantum particles (and their antiparticles) decay by the action of weak nuclear interaction [2]. This situation had as one of its sustenance the notorious validity of the $C P$ symmetry in processes conducted by other fundamental interactions: Strong nuclear and Electromagnetic [3, 4. That this was believed can be seen, for example, in the book "Quantum Mechanics" (QM) by Feynman [5], where it is discussed in accessible terms, both conceptually ${ }^{1}$ and mathematically, the neutral Meson $K$ (or Kaon). In Ref. [5], the $C P$ symmetry figure is noted when the author colloquially affirms that the neutral Kaon would remain "forever" in the $\left|K_{2}\right\rangle$ state $\left.\right|^{2}$ in the event that the only decay channel available is in two Pions. It was known that the neutral Kaon, when it was initially in the $\left|K_{2}\right\rangle$ state, could decay in three Pions preserving $C P$ symmetry.

The situation described in the paragraph above undergoes a change from the year 1964 with the experimental discovery of the breaking of $C P$ symmetry in the decay of the neutral Kaon into two Pions [7 9], a fact that Feynman's famous book does not incorporate because it was written before it. The partial outdating of Ref. [5] was even more evident after the discoveries, in 2001 and

\footnotetext{
* Correspondence email address: bulnes@unifap.br

1 Whereby, following [6], the neutral Kaon can be treated as a system of two quantum states, and thus corresponding to a twodimensional Hilbert space.

2 Of which it was obtained at time $t=0$.
}

2019, of new cases of breach of $C P$ symmetry in the decay of Mesons $B^{0}$ and $D^{0}$, respectively, [10 12].

On the other hand, it appears that the development presented in that chapter of Ref. [5] did not allow its author to determine a mathematical expression that could accommodate the mean lifetime for the "long" decay of the neutral Kaon into three Pions from $\left|K_{2}\right\rangle$ state. However, even in the context of $C P$ symmetry, it is possible to accommodate this second mean lifetime as long as a small break in CPT symmetry is assumed.

\subsection{Important and relevant aspects to be considered}

In this subsection we make a very small and simple review of some mathematical aspects related to the treatment of neutral Kaon and some comments regarding the development presented in Ref. [5], which will be useful in the following section. Let us start with the relevant vector basis for this description, here called $B_{S}$, $B_{C P}$ and $B_{W}$, as follows,

$$
B_{S}=\left\{\left|K_{0}\right\rangle,\left|\bar{K}_{0}\right\rangle\right\}
$$

formed by the eigenvectors of the so-called strangeness operator $^{3} \mathbf{S}$ [14. These vectors do not correspond to stationary states, as those studied in ordinary MQ, since the weak interaction makes the neutral Kaon to be unstable [15]. In addition to this base, we have,

$$
B_{C P}=\left\{\left|K_{1}\right\rangle,\left|K_{2}\right\rangle\right\}
$$

\footnotetext{
3 For particles sensitive to strong nuclear interactions containing in their internal structure the strange quark (s), the difference between the number of antiquarks "s" and the number of quarks "s" determines the so-called "strangeness quantum number" [13.
} 
which has the operator's eigenvectors $\mathbf{C P}$ product as its elements [14. Nor do these vectors correspond to stationary states due to the weak interaction [15]. It is important to say, within the context of validity of the $C P$ symmetry, that the $\left|K_{2}\right\rangle$ state is not compatible with the decay of the neutral Kaon into two Pions and the $\left|K_{1}\right\rangle$ state is incompatible with the decay of the same into three Pions. Finally, let's look at the base,

$$
B_{W}=\left\{\left|K_{S}\right\rangle,\left|K_{L}\right\rangle\right\}
$$

which incorporates the eigenvectors of the so-called Mass operator ${ }^{4} \mathcal{W}[14]$, suitable for describing the states in the situation of breaking $C P$ symmetry in the decay of the neutral Kaon into two Pions.

Now let's look at the relationships between the elements of those bases, those that allow the passage from one to another [15],

$$
\begin{aligned}
& \left|K_{1}\right\rangle=\frac{1}{\sqrt{2}}\left(\left|K^{0}\right\rangle+\left|\bar{K}^{0}\right\rangle\right) \\
& \left|K_{2}\right\rangle=\frac{1}{\sqrt{2}}\left(\left|K^{0}\right\rangle-\left|\bar{K}^{0}\right\rangle\right)
\end{aligned}
$$

and,

$$
\begin{aligned}
& \left|K_{S}\right\rangle=\left|K_{1}\right\rangle+\epsilon\left|K_{2}\right\rangle \\
& \left|K_{L}\right\rangle=\left|K_{2}\right\rangle+\epsilon\left|K_{1}\right\rangle
\end{aligned}
$$

$\epsilon$ being a parameter that characterizes the breaking of $C P$ symmetry.

Next, we should pay attention to the expression (11.44) of Ref. [5], which we copied below,

$$
\left\langle\bar{K}^{0}|\mathcal{W}| K^{0}\right\rangle=\left\langle K^{0}|\mathcal{W}| \bar{K}^{0}\right\rangle
$$

that Feynman presented under the argument that "matter and antimatter have exactly ${ }^{5}$ the same behavior". With the expression (8), the calculation related to the neutral Kaon decay time begins, in that reference. The mathematical relation (8), stated in concrete terms, results from: (i) the null value of the element $\mathcal{W}_{12}$ of the matrix of Mass $\mathcal{W}$ [14, and (ii) the acceptance of CPT theorem ${ }^{6}[14$. However, as in Ref. [5] these terms were not used during the presentation of its expression (11.44), it will be convenient to justify the wording in (i) and (ii).

\footnotetext{
${ }^{4}$ In the non-relativistic MQ, in which stable quantum particles are considered, the inclusion of any "Mass matrix" would be inconsistent, since the state of the particle, if expressed on the basis of the corresponding eigenvectors, would generally be written as a linear combination of the elements of this base, and thus would not have a defined mass, although the value of the mass of the particle would appear included in the Schrödinger equation.

5 Interpretation currently being reevaluated based on recent discoveries [16.

6 Of which the description of physical processes is considered invariant in relationship to the joint performance of $C, P$ and $T$ symmetry operations [1].
}

To begin, it is advisable to rewrite the $\mathcal{W}$ matrix on the $B_{S}$ base. Thus, it is found, in particular, that the element $\mathcal{W}_{12}$ is given by the expression,

$$
\mathcal{W}_{12}=\left\langle K_{1}|\mathcal{W}| K_{2}\right\rangle=\frac{1}{2}\left(\left\langleK^{0}\left|+\left\langle\bar{K}^{0}\right|\right) \mathcal{W}\left(\left|K^{0}\right\rangle-\left|\bar{K}^{0}\right\rangle\right)\right.\right.
$$

or,

$$
\begin{aligned}
\mathcal{W}_{12}= & \frac{1}{2}\left(\left\langle K^{0}|\mathcal{W}| K^{0}\right\rangle-\left\langle K^{0}|\mathcal{W}| \bar{K}^{0}\right\rangle\right. \\
& \left.+\left\langle\bar{K}^{0}|\mathcal{W}| K^{0}\right\rangle-\left\langle\bar{K}^{0}|\mathcal{W}| \bar{K}^{0}\right\rangle\right)
\end{aligned}
$$

On the other hand, for the neutral Kaon system the following relationship [14], is correct,

$$
\epsilon=\frac{\mathcal{W}_{12}}{m_{L}-m_{S}}
$$

$m_{S}$ e $m_{L}$ being the eigenvalues associated with the eigenvectors of the $B_{W}$ base. Thus, in the context in which the $C P$ symmetry is in effect $(\epsilon=0)$, we have,

$$
\mathcal{W}_{12}=0
$$

which, together with 10 , it follows $\mathrm{s}^{7}$ that,

$$
\left\langle K^{0}|\mathcal{W}| K^{0}\right\rangle-\left\langle\bar{K}^{0}|\mathcal{W}| \bar{K}^{0}\right\rangle=\left\langle K^{0}|\mathcal{W}| \bar{K}^{0}\right\rangle-\left\langle\bar{K}^{0}|\mathcal{W}| K^{0}\right\rangle
$$

Subsequently, as a consequence of the assumed CPT invariance for the considered decay processes, it must be fulfilled that [14,

$$
\left\langle K^{0}|\mathcal{W}| K^{0}\right\rangle=\left\langle\bar{K}^{0}|\mathcal{W}| \bar{K}^{0}\right\rangle
$$

Thus, from expressions (13) and (14) the following is obtained,

$$
\left\langle\bar{K}^{0}|\mathcal{W}| K^{0}\right\rangle=\left\langle K^{0}|\mathcal{W}| \bar{K}^{0}\right\rangle
$$

which is the very expression (11.44) of Ref. [5]. Therefore, 15 is a consequence of the rule of $C P$ symmetry in the decay processes considered in Ref. [5].

In relation to the mathematical development presented in Ref. [5], we can see the intention to keep it simple and note that, in solving equations (11.47) in Ref. [5], keep the terms $E_{0} C_{+}$and $E_{0} C_{-}$, redefined 8 (as was done) or joining them with the complex terms $A C_{+}$and $A C_{-}$, respectively, would result in the same expression. Considering the physical context and mathematical development established in Ref. [5], the author was unable to generate an expression to accommodate the mean lifetime (with finite value) for the neutral Kaon in the situation in which, being in $t=0$ in the $\left|K_{2}\right\rangle$ state, decays into three Pions.

Note that, in the context of the MQ, that colloquial comment by Feynman of the permanence "forever" of

\footnotetext{
${ }^{7}$ Note that the validity of $C P$ symmetry does not imply null values for both members in 11 .

8 Through a displacement of the origin on the energy scale.
} 
the neutral Kaon in the $\left|K_{2}\right\rangle$ state means, if taken "to the letter", that it would behave as a steady state; which would only make sense if it were possible to "turn off" the weak interaction to "deactivate" the decay channe into three Pions. This is the danger of this comment: it creates confusion among some readers.

In this article, considering only the neutral Kaon decays that preserve $C P$ symmetry and partially taking into account what was developed in Ref. [5], we have built a complementary solution to this development, "reasonably" simple consistent with finite decay times.

\section{Variant to the Feynman solution}

We start here trying to understand why the equations (11.47) of Ref. [5] do not generate mathematical expressions that allow to fit decay times for the neutral Kaon with finite values. Let's look at these equations ${ }^{11}$ which we copied below, as in a "mathematical tests" laboratory. We have,

$$
\begin{aligned}
& i \hbar\left(\frac{d C_{+}}{d t}\right)=E_{0} C_{+}+A C_{-}+A C_{+} \\
& i \hbar\left(\frac{d C_{-}}{d t}\right)=E_{0} C_{-}+A C_{+}+A C_{-}
\end{aligned}
$$

When we keep $E_{0} C_{+}$and $E_{0} C_{-}$in the above equations, joining them with the complex terms $A C_{+}$and $A C_{-}$, respectively, we have these two complex numbers, $A_{3}$ and $A_{4}$, with,

$$
A_{3}=A, \quad e \quad A_{4}=\left(E_{0}+\operatorname{Re}(A)\right)+i \operatorname{Im}(A)
$$

Note that $A_{3}$ and $A_{4}$ have the same imaginary part, and, precisely, this is the problem. It is simple to see that the two equations above, (16) and (17), include two parameters: the real and the imaginary part of the complex number $A$, so that they describe, in Ref. [5], the probability amplitude that the neutral Kaon, when it was in the $\left|K_{1}\right\rangle$ state at $t=0$, remains in that state for any $t>0$, leaving no free parameters that can describe the situation in which the neutral Kaon, being in the $\left|K_{2}\right\rangle$ state for $t=0$, it decays into three Pions. It seems clear that we need two more parameters.

The previous one leads us to pay attention to the following situation: In the case of having, instead of equations (16) and (17), two of the following type,

$$
\begin{gathered}
i \hbar\left(\frac{d C_{+}}{d t}\right)=A_{1} C_{-}+A_{2} C_{+} \\
i \hbar\left(\frac{d C_{-}}{d t}\right)=A_{1} C_{+}+A_{2} C_{-}
\end{gathered}
$$

\footnotetext{
${ }^{9}$ Explicitly disregarded in Ref. 5].

10 The complete and exact solution can be seen, for example, in [17].

11 The meaning of each of the symbols that appears on them can be found in Ref. 5 .
}

being $A_{1}$ and $A_{2}$ complex numbers that do not have any of the four equal parts, then we would no longer have two, but four real parameters available and we would possibly find the type of solution sought. Let's look at this. It can be easily shown that, following the procedure presented in Ref. [5], starting with equations (11.47), but now replaced by our equations (18) and (19), until arriving at equations (11.51) of that reference, now modified, the corresponding solutions are,

$$
\begin{aligned}
& C_{1}(t)=C_{1}(0) \exp \left\{-i\left(A_{1}+A_{2}\right) t\right\} \\
& C_{2}(t)=C_{2}(0) \exp \left\{-i\left(A_{1}-A_{2}\right) t\right\}
\end{aligned}
$$

with $C_{1}=1 / \sqrt{2}\left(C_{+}+C_{-}\right)$and $C_{2}=1 / \sqrt{2}\left(C_{+}-C_{-}\right)$, Ref. [5]. Now we see clearly, and not just in a qualitative way, why it was not possible to find in Ref. [5] a second solution that can be linked with a finite mean lifetime: In the mathematical expression (21), when $C_{2}(0)=1$ and $A_{1}=A_{2}$, we get $C_{2}(t)=1$, as in Ref. [5] ; but, in the case of terms: $A 1 \neq A 2$, then the amplitude $C_{2}(t)$ decays exponentially with time, as expected.

After discovering which of the mathematical characteristic is absent in equations 16 and $(17)$, that would allow us to arrive at the expressions that could be linked with the finite mean lifetime corresponding to the decay of the neutral Kaon, let's see if there are physical arguments that could bring us equations of type 18 and $(19)$.

It is evident that some important changes will have to be made in relationship to the physical context assumed in Ref. [5], as it is not possible to find two distinct complex numbers $A_{1}$ and $A_{2}$, as we wish, from the expression (11.44) of Ref. 5, here copied in expression (15), which, as already mentioned, is the starting point to the calculation linked to the mean lifetime of the neutral Kaon decay of this reference.

Going back to our subsection 1.1 we see that, within the context of the $C P$ symmetry and assuming a small break ${ }^{12}$ in the $C P T$ symmetry, so that we can write, instead of (14), the following expression,

$$
\left\langle K^{0}|\mathcal{W}| K^{0}\right\rangle \approx\left\langle\bar{K}^{0}|\mathcal{W}| \bar{K}^{0}\right\rangle
$$

we would have a mathematically favorable condition to accommodate two finite mean lifetime, and not just one, because, as a consequence of 22 and $(13)$, the following expression would result, instead of 15 ,

$$
\left\langle\bar{K}^{0}|\mathcal{W}| K^{0}\right\rangle \approx\left\langle K^{0}|\mathcal{W}| \bar{K}^{0}\right\rangle
$$

$C P$ symmetry being effectively preserved, since the above expressions are such that they do not alter the equality 13. If we now write,

$$
\begin{aligned}
& \left\langle\bar{K}^{0}|\mathcal{W}| K^{0}\right\rangle=A_{1} \\
& \left\langle K^{0}|\mathcal{W}| \bar{K}^{0}\right\rangle=A_{2}
\end{aligned}
$$

\footnotetext{
12 Subject that was analyzed, in 1965, by Bell and Steinberger [5].
} 
the complex numbers $A_{1}$ and $A_{2}$ being necessarily different, but "neighbor:13,', since we are assuming a small break in the $C P T$ symmetry. When we incorporate these numbers in the development given in Ref. [5], replacing the parameter $A$ considered there by them, and making the necessary adaptations, it is simple to show, following the same sequence of steps given in Ref. [5], beginning by its expression (11.44), which will then have the aspect of our expressions (24) and (25), which obtain modifications to the equations (11.47) as given here in (18) and 190 , where the terms $E_{0} C_{+}$and $E_{0} C_{-}$can be disregarded or combined with the complex terms $A C_{+}$and $A C_{-}$, respectively. For what follows, it is advisable to explicitly write the complex numbers $A_{1}$ and $A_{2}$,

$$
\begin{aligned}
& A_{1}=\alpha_{1}-i \beta_{1} \\
& A_{2}=\alpha_{2}-i \beta_{2}
\end{aligned}
$$

Now, considering that the two initial conditions $(t=0)$ considered for the neutral Kaon in Ref. [5] were: $(i)$ that it is in the $\left|K_{1}\right\rangle$ state, that is, $C_{1}(0)=1$ and $C_{2}(0)=0$, or, (ii) that is in the $\left|K_{2}\right\rangle$ state, that is, $C_{1}(0)=0$ and $C_{2}(0)=1$. In particular, for the probability amplitude that the neutral Kaon is, in an instant $t>0$, in the same $\left|K_{1}\right\rangle$ state, we have the expression,

$$
C_{1}(t)=\exp \left\{-\left(\beta_{1}+\beta_{2}\right) t\right\} \exp \left\{-i\left(\alpha_{1}+\alpha_{2}\right) t\right\}
$$

and for the corresponding probability,

$$
P_{1}(t)=\left|C_{1}(t)\right|^{2}=\exp \left\{-2\left(\beta_{1}+\beta_{2}\right) t\right\}
$$

and the mean lifetime is equal to,

$$
\tau_{1}=1 /\left(2\left(\beta_{1}+\beta_{2}\right)\right)
$$

In addition, contrary to the result found in Ref. [5], for the decay of the neutral Kaon in the situation where it is at the moment $t=0$ in the $\left|K_{2}\right\rangle$ state, we have, for $t>0$ and according to equations 20 and 21 , the following probability amplitudes 14

$$
\begin{gathered}
C_{1}(t)=0 \\
C_{2}(t)=\exp \left\{-\left(\beta_{2}-\beta_{1}\right) t\right\} \exp \left\{-i\left(\alpha_{2}-\alpha_{1}\right) t\right\}
\end{gathered}
$$

and, in particular, the corresponding probability of finding the neutral Kaon in the same $\left|K_{2}\right\rangle$ state, at time $t>0$, is equal to,

$$
P_{2}(t)=\left|C_{2}(t)\right|^{2}=\exp \left\{-2\left(\beta_{2}-\beta_{1}\right) t\right\}
$$

and the mean lifetime is equal to,

$$
\tau_{2}=1 /\left(2\left(\beta_{2}-\beta_{1}\right)\right)
$$

\footnotetext{
13 We can imagine them as two very close vectors, but not parallel, in the complex plane.

${ }^{14} C_{1}$, that the neutral Kaon is found in an instant $t>0$ in the $\left|K_{1}\right\rangle$ state and, $C_{2}$, that it is found in the $\left|K_{2}\right\rangle$ state.
}

Instead, according to Ref. [5], the probability of finding the neutral Kaon in the $\left|K_{2}\right\rangle$ state, for any instant $t>0$, does not depend on time and is equal to $P_{2}(t)=1$; that is, in the context considered, the Kaon "does not decay".

To conclude this section, let's look at some relationships between the parameters. Considering that the decay with an mean lifetime $\tau_{2}$ occurs $\lambda$ times more slowly than the first one (with an mean lifetime $\tau_{1}$ ), we have that $\tau_{1}=(1 / \lambda) \tau_{2}$, from which we write,

$$
\beta_{2}-\beta_{1}=\frac{1}{\lambda}\left(\beta_{1}+\beta_{2}\right)
$$

or equivalently,

$$
\beta_{2}=\frac{\lambda+1}{\lambda-1} \beta_{1}
$$

Also, like $\tau_{1}=10^{-10} \mathrm{~s}$, Ref. [5], and from (30), we have,

$$
\beta_{1}+\beta_{2}=0.5 \times 10^{10} s .
$$

Hence, from 35 and 36 , it follows that,

$$
\begin{aligned}
& \beta_{1}=0,25\left(\frac{\lambda-1}{\lambda}\right) \times 10^{10} \approx 0,2495 \times 10^{10} \\
& \beta_{2}=0,25\left(\frac{\lambda+1}{\lambda}\right) \times 10^{10} \approx 0,2504 \times 10^{10}
\end{aligned}
$$

using the approximate value $\lambda=600$, consistent with the experimental data [5].

Note that the solution considered here excludes the possibility of having any information regarding the parameters $\alpha_{1}$ and $\alpha_{2}$ as well as their relationship with the other parameters; in this sense, our solution is approximate ${ }^{15}$ In order to have some information about these parameters, we would have to consider an additional condition to the approach made; for example, that the complex numbers $A_{1}$ and $A_{2}$, although distinct, have the same module. In that case,

$$
\alpha_{1}^{2}+{\beta_{1}}^{2}=\alpha_{2}^{2}+{\beta_{2}}^{2}
$$

And using 35 we obtain,

$$
\begin{gathered}
\alpha_{1}^{2}+\beta_{1}^{2}=\alpha_{2}^{2}+\left(\frac{\lambda+1}{\lambda-1}\right)^{2} \beta_{1}^{2} \\
\alpha_{1}^{2}-\alpha_{2}^{2}=\frac{4 \lambda}{(\lambda-1)^{2}} \beta_{1}^{2} \\
\alpha_{1}^{2}-\alpha_{2}^{2}=\frac{2}{\lambda} \times 10^{20}
\end{gathered}
$$

In the expression above, the limit $\lambda \rightarrow \infty$ implies $\alpha_{1}=$ $\alpha_{2}$, which in turn implies, using (34), that $\beta_{2}=\beta_{1}$, consistently with the context presented in Ref. [5]. As might be expected, the transition from our solution to that in Ref. [5] is controlled only by the value of the parameter $\lambda$.

\footnotetext{
15 It is characteristic of any approximate solution to carry less information than that contained in the exact corresponding solution.
} 


\section{Conclusion}

In this article, a complementary solution to the one given in Ref. [5] was constructed for the problem of determining expressions that can accommodate the mean lifetime of the neutral Kaon in its decay through two independent channels that preserve the $C P$ symmetry. Unlike the absence of an expression that allows to accommodate the mean lifetime for the decay of the neutral Kaon when it was initially at $(t=0)$ in the $\left|K_{2}\right\rangle$ state in Ref. [5], here we build it. It was shown that this complementary solution is compatible with a physical context in which the CPT symmetry is affected by a "small" break, in the sense that the relationship (22) is valid, and the $C P$ symmetry is in effect. Finally, in addition to the reason already mentioned in the previous section for considering our solution as an approximate one, there is a second argument for such consideration: so far everything indicates that CPT symmetry is preserved in the processes conducted by fundamental physical interactions.

\section{References}

[1] J.L. Rosner, Braz. J. Phys. 32, 147 (2001).

[2] D. Bailin, Weak Interactions (Adam Hilger, Bristol, 1982).

[3] R.D. Peccei and H.R. Quinn, Phys. Rev. Lett. 38, 1440 (1977).

[4] R.D. Peccei, arXiv:hep-ph/9807516v1 (1998).

[5] R.P. Feynman, R.B. Leighton and M. Sands, The Feynman Lectures on Physics (Addison-Wesley, Mass. 1964), v. III.

[6] M. Gell-Mann, A. Pais, Phys. Rev. 97, 1387 (1955).

[7] J.H. Christenson, J.W. Cronin, V.L. Fitch and R. Turlay, Phys. Rev. Lett. 13, 138 (1964).

[8] J.W. Cronin, Nobel lecture (1980).

[9] V.L. Fitch, Nobel lecture (1980).

[10] B. Aubert, D. Boutigny, J.M. Gailard, A. Hicheur, Y. Karyotakis, J.P. Lees, P. Robbe, V. Tisserand, A. Palano, G.P. Chen et al., Phys. Rev. Lett. 87, 091801 (2001).

[11] K. Abe, K. Abe, R. Abe, I. Adachi, Byoung Sup Ahn, H. Aihara, M. M. Akatsu, G. Alimonti, K. Asai, M. Asai et al., Phys. Rev. Lett. 87, 091802 (2001).

[12] R. Aaij, C. Abellán Beteta, B. Adeva, M. Adinolfi, C.A. Aidala, Z. Ajaltouni, S. Akar, P. Albicocco, J. Albrecht, F. Alessio et al., Phys. Rev. Lett. 122, 211803 (2019).

[13] B. Povh, K. Rith, C. Scholz and F. Zetsche, Particles and Nuclei. An introduction to the physical concepts (Springer-Verlag, Berlin, 1995).

[14] O. Nachtmann, Elementary Particle Physics. Concepts and Phenomena (Springer-Verlag, Berlin, 1990).

[15] K. Gottfried and V.F. Weisskopf, Concepts of Particle Physics (Clarendon Press, 1984), v. 1.
[16] https://news.syr.edu/blog/2019/03/27/physicists-re veal-why-matter-dominates-the-universe, accessed in Apr. 2021.

[17] W. Greiner and B. Müller, Gauge Theory of Weak Interactions (Springer-Verlag, Berlin, 2000).

[18] J.S. Bell and J. Steinberger, Proceedings of the Oxford International Conference on Elementary Particles, 2985 (1968). 